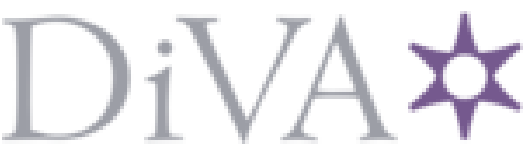

http://www.diva-portal.org

Postprint

This is the accepted version of a paper presented at International Workshop on Enterprise Distributed Object Computing, EDOCW.

Citation for the original published paper:

Wester, P., Heiding, F., Lagerström, R. (2021)

Anomaly-based Intrusion Detection using Tree Augmented Naive Bayes

In: Australia: IEEE

N.B. When citing this work, cite the original published paper.

Permanent link to this version:

http://urn.kb.se/resolve?urn=urn:nbn:se:kth:diva-305880 


\title{
Anomaly-based Intrusion Detection using Tree Augmented Naive Bayes
}

\author{
Philip Wester, Fredrik Heiding, and Robert Lagerström \\ School of Electrical Engineering \& Computer Science \\ KTH Royal Institute of Technology \\ Stockholm, Sweden
}

\begin{abstract}
Information technology is continuously becoming a more central part of society and together with the increased connectivity and inter-dependency of devices, it is becoming more important to keep systems secure. Most modern enterprises use some form of intrusion detection in order to detect hostile cyber activity that enters the organization. One of the major challenges of intrusion detection in computer networks is to detect types of intrusions that have previously not been encountered. These unknown intrusions are generally detected by methods collectively called anomaly detection. It is nowadays popular to use various artificial intelligence schemes to enhance anomaly detection of network traffic, and many state-of-the-art models reach a detection rate of well over $99 \%$. One such promising algorithm is the Tree Augmented Naive Bayes (TAN) Classifier. However, it is crucial to implement TAN correctly in order to benefit from its full performance. This study implements a TAN classifier for anomaly based intrusion detection of computer network traffic, and displays practical insights on how to maximize its performance. The algorithm is implemented in two data sets with data from simulated cyber attacks: NSL-KDD and UNSW-NB15. We contribute to the field by validating the usefulness of TAN for anomaly detection in computer networks, as well as providing practical insights to new practitioners who want to utilize TAN in intrusion detection systems.
\end{abstract}

Index Terms-Intrusion detection, IDS, Anomaly detection, Tree Augmented Naive Bayes, TAN, Machine learning, Network based intrusion detection.

\section{INTRODUCTION}

Cyber security is continuously becoming a more important concern for organizations, the recent flood of enterprise oriented cyber attacks is a keen reminder of the price of insufficient cyber security [1], [2], [3]. In addition to the last wave of attacks, recent years have brought even more devastating attacks such as severe botnet attacks targeting both infrastructures and organizations [4], as well as the Petya ransomware attack that affected users and organizations in more than 60 countries and is estimated to have cost Maers alone 300m USD [5].

Thus, organizations are motivated to maintain adequate levels of cyber security, but this can be a daunting task. Writing and validating software in a secure manner is both expensive and complicated. The often fast-paced environment of modern enterprise architectures makes it difficult to stay up to date with the required cyber security standards. Modern systems and software are undergoing continuous updates and are interconnected with a large number of different tools, protocols, and services. These tools, protocols, and services are also being updated and changed continuously, while new ones are being introduced regularly, constituting a complicated and unpredictable environment. With this foundation vulnerabilities are bound to occur [6].

Because of this, it is important to use adequate technologies to identify and prevent ongoing attacks. Today, intrusion detection systems (IDS) are a fundamental part of the security of any small- to large scale system [7]. By monitoring the network traffic of an organization for anomalies or traffic with known malicious signatures, it is possible to prevent an attack before it has time to gain momentum.

Intrusion detection can be divided into two methods, signature detection and anomaly detection. Signature detection includes methods where known attacks are identified by information stored in the system, so called "signatures". Anomaly detection is based on categorizing predefined notions of what "normal" traffic is, then classifying everything outside of that category as "anomalies" or potential intrusions. Signature detection is effective against the known attacks in its data base, but it is unable to detect unknown intrusions. Anomaly detection might detect new types of intrusions if trained correctly, but runs a higher risk of creating false positives (classifying legitimate traffic as malicious). Anomaly detection also tends to have a lower true positive rate (correctly identifying malicious traffic as malicious) than signature detection for known attacks. It is generally accepted that a good intrusion detection system should rely on both signature detection and anomaly detection [8], [9].

Because of the triviality and high effectiveness of modern signature detection methods, most contemporary research of intrusion detection has focused on improving methods of anomaly detection. Unfortunately, anomaly detection in computer networks is not always straight forward, as some attacks may appear very similar to ordinary traffic [7]. In order to increase the chances of detecting malicious network traffic, anomaly based intrusion detection often use AI-enhanced schemes for their predictions. As further discussed in Section III and VI, much research has been done on such AI-enhanced network intrusion detection, and some state-of-the-art algorithms achieve high levels of accuracy, sometimes well above $99 \%$ ( [10], [11], [12]). 
One of the promising algorithms is Tree Augmented Naive Bayes (TAN). Previous implementations of TAN based intrusion detection have achieved on par with other state-of-the-art algorithms, reaching above 99\% accuracy ( [13], [12], [14]). Because TAN is a promising solution to enhance the prediction accuracy of network intrusion detection, organizations may be tempted to implement the algorithm in order to enhance their cyber security. This is good, however, it is important that some factors are taken into consideration while implementing TAN.

In this study we have implemented a TAN classifier for Anomaly-based intrusion detection. Partly in order to further validate its accuracy and usefulness for anomaly based intrusion detection, partly in order to clarify how to implement TAN in order to maximize its performance.

The implemented TAN classifier is based on the one proposed by Friedman et al. in [15], and used with smoothing (as is standard for TAN classifiers [16]) to achieve sufficient accuracy. The algorithm is implemented on the NSL-KDD and the UNSW-NB15 data sets, further described in Section II-B. It is worth to note that anomaly detection is a large field of study present in many domains. In this study we are only concerned with anomaly detection in network traffic, trying to categorize malicious network data and distinguish it from ordinary traffic.

The purpose of the study is thus to provide practical insights into the usefulness of TAN for anomaly detection in computer networks, as well as what to be aware of when implementing TAN. By doing so, we hope to contribute to the community both by adding practical insights in the implementation of the algorithm, as well as confirming and contradicting previous reports on the performance of TAN.

The conclusion of the paper is that our TAN implementation performs consistently with previous research and is able to classify malicious traffic with more than $99 \%$ accuracy. However, the implementation settings are crucial in order to achieve adequate performance.

\section{A. Outline}

The remainder of the article is structured as follows: Section II describes relevant information for readers who are not too familiar with the area. Section III discuss the current state of the research field. Section IV describes our Python based implementation of a Tree Augmented Naive Bayes Classifier for intrusion detection. Section V presents the results obtained by our TAN classifier, more specifically how well it managed to identify, classify and categorize malicious and normal traffic. Section VI discuss the performance of the model, the choice of algorithms, the general validity of our method, and our choice of data sets, and Section VII concludes the article.

\section{Theoretical Preliminaries}

This Section gives a brief introduction to background information for relevant areas of the study. Section II-A explains the fundamentals of using Bayesian networks and Tree

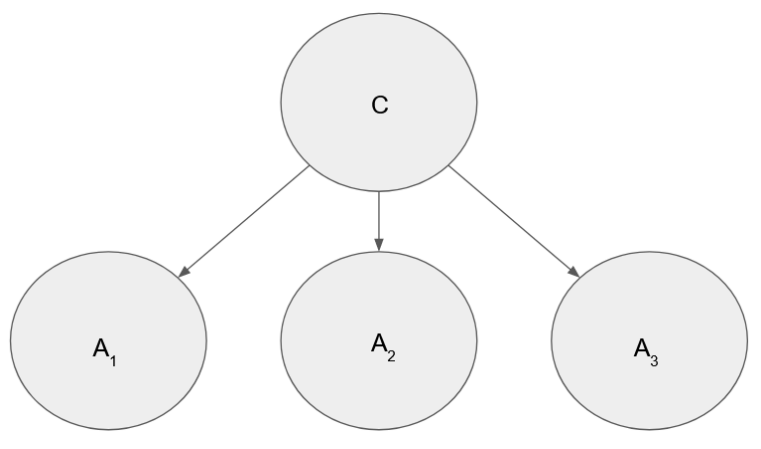

Fig. 1. Naive Bayes Structure

Augmented Naive Bayes as classifiers. Section II-B gives information about the data sets and explains the methods used for data preparation.

\section{A. Bayesian Networks}

This subsection aims to clarify parts of Naive Bayes and Tree Augmented Naive Bayes, which are needed in order to understand the rest of the work.

1) Naive Bayes Classifier: The Naive Bayes Classifier uses a Bayesian Network to classify one or more data sets. Given a data set with the attributes $A$, the Bayesian network consists of two types of nodes: the class node $C$ and the attribute nodes $A_{i}$, where $0<i \leq|A|$. There are $|A|$ number of edges, one between $C$ and each $A_{i}$, as can be seen in figure 1 . The conditional probability $P\left(a_{i} \mid c\right)$ for each possible class $c \in C$, attribute value $a_{i} \in A_{i}$, and attribute $A_{i} \in A$ are calculated from the training data set. For a given test data instance $A^{T e s t}$, the predicted class is the class $c \in C$ that maximizes the probability for the given attributes, thus the class $c$ that maximizes the probability $P\left(a_{0}^{T e s t}, a_{1}^{T e s t}, \ldots, a_{n}^{T e s t} \mid c\right)$ [15].

The Naive Bayes Classifier is somewhat flawed in its fundamental assumption that all parameters are conditionally independent. This is can be an issue when the classifier is applied to real world scenarios, as most parameters depend on other parameters, at least to some extent. One way to solve this problem is to introduce conditional dependency, which is what Friedman et al. proposed with the Tree Augmenter Naive Bayes classifier [15].

2) Tree Augmented Naive Bayes Classifier: The Tree Augmented Naive Bayes (TAN) classifier extends the Naive Bayes classifier by allowing conditional dependencies between the edges of the tree. This means that the TAN networks does not only contain edges between the class node $C$ and the attributes in $A$, but also edges between the different attributes in $A$, as can be seen in figure 2 . 


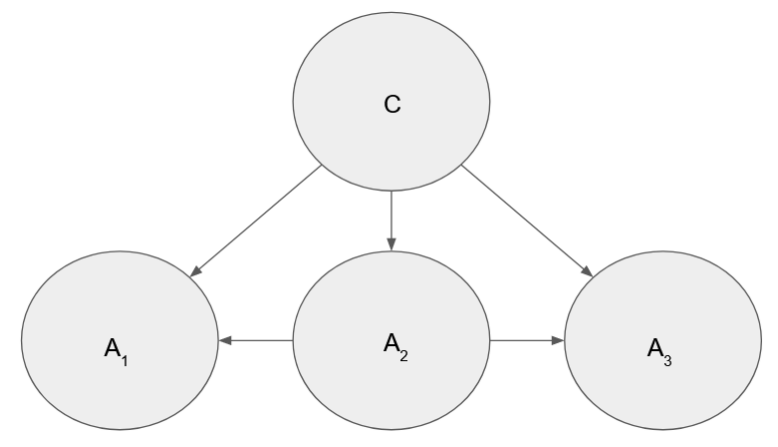

Fig. 2. TAN Structure

In order for TAN to run in polynomial time, each attribute node $A_{i}$ can have at most one attribute $A_{j}(j \neq i)$ as parent. There is no restriction on children, meaning that an attribute $A_{i}$ still can have multiple outgoing edges. To decide the structure of the TAN network, Friedman et al. adapts the algorithm proposed by Chow et al. for tree construction [17] to calculate the conditional mutual information between all attributes and then construct a maximum spanning tree where a root node $A_{r}$ is chosen. In the case of our network traffic, the classes are either normal or malicious traffic, or more specific attack type classes such as DoS attacks or probe attacks. The attributes are traffic characteristics such as which service is used (HTTP, telnet, FTP etc.) or the source IP of the traffic, further described in Section II-B.

When calculating the conditional probabilities in TAN, the data is partitioned based on possible values of the conditionals. As each TAN attribute depends on both the class node $C$ and its parent attribute node $p a\left(A_{i}\right)$, the number of partitions in TAN are at least twice as large as with classic Naive Bayes classification. Therefore it is possible that some partitions in TAN contain very few instances, especially in small data sets, which makes the conditional probability unreliable. The probability may even be incorrectly set to zero, leading to the so called zero frequency problem. A simple way to counter this is to replace the zero probability with a small constant, but many more sophisticated smoothing techniques have been proposed over the years, such as the Laplace smoothing [16] or the smoothing operator proposed by Friedman et al. in [15] and displayed in equation 2.

Without the smoothing operator, the probability score for a TAN classification $c$ can be calculated as:

$$
P\left(c \mid A^{\text {test }}\right)=P(c) P\left(a_{r}^{\text {test }} \mid c\right) \prod_{i=1, i \neq r}^{n} P\left(a_{i}^{\text {test }} \mid c, p a\left(a_{i}^{\text {test }}\right)\right)
$$

where $p a\left(a_{i}\right)$ is the value of the parent attribute of $A_{i}$ [18].

With smoothing, TAN can be calculated with:

$$
P\left(a_{i} \mid c, p a\left(a_{i}\right)\right)=\frac{N \cdot \hat{P}\left(p a\left(a_{i}\right), c\right) \cdot \hat{P}\left(a_{i} \mid c, p a\left(a_{i}\right)\right)}{N \cdot \hat{P}\left(p a\left(a_{i}\right), c\right)+N^{0}}+\frac{N^{0} \cdot \hat{P}\left(a_{i}\right)}{N \cdot \hat{P}\left(p a\left(a_{i}\right), c\right)+N^{0}}
$$

where $N$ is the size of the data set and $N^{0}$ is a constant [15].

\section{B. Data sets}

The project used two different data sets containing data of malicious and normal network traffic: NSL-KDD [19] and UNSW-NB15 [20]. They are presented below along with descriptions of how the data sets were adapted for use.

1) Discretization: One of the primary challenges of using real world data on a TAN network is the fact that TAN treats discrete values while the data we want to analyze may be continuous. To counter this, one can discretize the continuous data before applying TAN. Two such discretization methods are equal width binning [21] and mdlp [22].

Equal width binning divides the data in intervals of equal size. This means that if a data set $D$ is to be discretized into $k$ sets, each set would have a size $\frac{x_{\max }-x_{\min }}{k}$, where $x_{\max }$ and $x_{\text {min }}$ represent the largest and the smallest values in $D$. The obvious flaw of equal width binning is its independence from the distribution of values $x \in D$. Because of this, a data set that is not uniformly distributed is bound to lose significant portions of information [21].

The mdlp method for discretization counters the potential information loss present in equal width binning by calculating where to split the data set to achieve the highest information gain (the information gain can be seen as the entropy loss when dividing the interval). This is done by analyzing the distribution of values and their classifications. The mdlp method thus creates a more precisely measured discretization of the data, as the intervals for discretization are more closely related to the similarities and dissimilarities in the original data set. This study used the mdlp method for discretization. A more detailed description of the mdlp discretization algorithm can be found in [22].

2) NSL-KDD: The KDD CUP ' 99 data set contains simulated traffic and intrusions from a military network environment. Although useful, the data set has received criticism as discussed by McHugh in [23]. The NSL-KDD data set is a reduction of the KDD CUP' 99 data set, where certain records were removed to make the data better reflect real world traffic. The improvements mainly consisted in minimizing the bias from the training set and improve distributions between the data sets. The data sets in NSL-KDD that this project used were:

- KDDTest+ containing 22544 records

- KDDTrain+ containing 125973 records

The data sets contain 24 different attack types which can be further divided into binary classification (normal/malicious) or 5 groups:

1) Normal traffic.

2) Probes: information gathering, ex. a TCP syn scan.

3) DoS (Denial of Service): An attempt to disrupt a service.

4) r2l (remote to local): An attack in attempt to gain access to a local machine, ex. Anonymous ftp login. 
5) $\mathbf{u 2 r}$ (user to root): A privilege escalation attack, ex. Exploitation of suoders files.

The data in itself is computer network traffic. The traffic is quantified with attributes such as protocol type (tcp,udp, icmp), service (http, telnet, ftp, ssh etc.), and number of failed logins [24].

3) UNSW-NB15: The UNSW-NB15 data set also contains normal and malicious computer network traffic and aims to be a modern representation of digital footprints. In this study, a prepared partition of the UNSW-NB15 data set (called "UNSW_NB15_training-set.csv" 1 and " UNSW_NB15_testing-set.csv" 2) was used. The training and test sets contain 175,341 and 82,332 records respectivly. There are 10 classes in the data set: Normal, Reconnaissance, Backdoor, DoS, Exploits, Analysis, Fuzzers, Worms, Shellcode, and Generic, as well as the binary classification of intrusion/normal traffic [25]. The traffic is quantified with attributes like those of the NSL-KDD data set, such as protocol type (tcp,udp, icmp, other) and service (http, telnet, ftp, ssh etc.). Nour et al. gives a thorough description of the UNSW-NB15 data set in [25].

\section{RELATED WORK}

Andersson et al. made an early introduction to intrusion detection and defined an intrusion threat as "The potential possibility of a deliberate unauthorized attempt to: (a) access information (b) manipulate information (c) render a system unreliable or unusable" [7]. The field has later expanded into multiple subcategories, including AI-enhanced intrusion detection.

There exist many studies on both anomaly based intrusion detection and AI-enhanced anomaly based intrusion detection [26]. Bhuyan et al. made a survey of state-of-the-art techniques for anomaly based intrusion detection systems and categorizes them in six groups: statistical, classification-based, clustering and outlier-based, soft computing, knowledge-based, and combination learners [26]. The study emphasized two distinguished criteria for classifying and evaluating intrusion detection schemes: detection strategy and evaluation of data sets. It is essential to present a systematic strategy for the anomaly detection, including convenient metrics for the performance measurement, such as prediction accuracy, detection rate and false positive rate. It is equally important to test the intrusion detection technique on a large and well known data set, in order to facilitate comparison with other studies and to facilitate the comparison of future studies with our study. Many of the analyzed detection schemes has around $90 \%$ accuracy, some have around $80 \%$ and below and others reach well over $90 \%$.

Hodge et al. made a similar survey, and categorized anomaly based detection in four segments: statistical methods, neural

\footnotetext{
${ }^{1}$ https://www.kaggle.com/mrwellsdavid/unsw-nb15?select=UNSW_NB15_ training-set.csv

${ }^{2}$ https://www.kaggle.com/mrwellsdavid/unsw-nb15? select=UNSW_NB15_ testing-set.csv
}

networks, machine learning methods, and hybrid systems (a combination of some of the other three categories). The study concludes that previous authors have implemented a wide range of techniques for anomaly detection, covering statistical, neural, machine learning, and hybrid techniques. No single method is universally applicable for anomaly detection, but each model may be suitable in different circumstances. Also, the study explains that they can not cover all possible approaches of anomaly detection, and encourages further research and implementation of anomaly detection schemes [27].

Similarly, we do not expect to give a full account of all subcategories and proposals of anomaly based intrusion detection in this Section. Rather, we present a selection of the state-ofthe-art solutions in order to give the reader a better understanding of our implementation. Thus, both studies on Naive Bayes based intrusion detection and intrusion detection using other schemes are presented. The studies vary in how many figures they present their accuracy values and detection rates with. Because of this, the summarized detection rates and accuracies given below may differ in terms of how many figures they are described with. Furthermore, some studies present the accuracy of models while others present the detection rate (many present both), in the case only one is presented, that is the one shown below. Therefore, the summarized studies are sometimes displayed with detection rates and sometimes with accuracy.

\section{A. Neural Networks for Anomaly Detection}

Ghosh et al. performed an early study using Neural Networks for intrusion detection in 1999. The study used labeled data and initialized multiple different networks with multiple different initial weights. The weights were adjusted with back propagation during the training phase. The network that gave the highest accuracy on the test data was picked for the results. The system achieved up to a $90.9 \%$ true positive rate with a false positive rate of $18.7 \%$. The false positive rate could be reduced to $0.01 \%$, but that would also decrease the accuracy to between $60-70 \%$. The study further found a considerable difference in detection accuracy for different attack types. The discovery rate of novel DoS (Denial of Service) attacks was roughly $20 \%$ and the rate of detecting new remote to local attacks was less than $10 \%$. Thus, showing that novel denial of service and remote to local attacks could not be discovered reliably [28].

Zhiqiang et al. used another neural networks scheme for anomaly based intrusion detection. A Deep Learning model using a feed-forward neural network was implemented on the UNSW-NB15 data set, achieving a prediction accuracy of $99.5 \%$ and a false positive rate of $0.47 \%$ [10].

Ding et al. used a Convolutional Neural Networks scheme to enhance anomaly based intrusion detection. The scheme was trained and tested using the NSL-KDD data set. The group also implemented two traditional machine learning methods 
(Random Forest and Support Vector Machines) as well as two deep learning methods (Belief Networks and Long Short Term Memory) to compare the results with that of their Convolutional Neural Network. The Convolutional Neural Network received a detection rate of $96.7356 \%$ for normal traffic, $85.2681 \%$ for DoS attacks, $73.9776 \%$ for probes, $17.7816 \%$ for $\mathrm{r} 2 \mathrm{l}$, and $8.9552 \%$ for $\mathrm{u} 2 \mathrm{r}$. The prediction rate for the other algorithms were lower in all cases. The false positive rate for the Convolutional Neural network scheme was $29.5098 \%$ for normal traffic, $1.9291 \%$ for DoS attacks, $1.7194 \%$ for probes, $0.2746 \%$ for R2L, and $0.0044 \%$ for R2L. The false positive rates for the other algorithms were generally higher [29].

\section{B. Various machine learning and decision tree schemes for anomaly detection}

Elsner et al. developed an unsupervised machine learning model to detect anomalies within enterprise applications. The model was based on a density-based clustering algorithm and achieved a detection accuracy of up to $90 \%$ [30].

Khan et al. proposed a method for intrusion detection using Clustering Trees and Support Vector Machines (CT-SVM). The method performed well in terms of accuracy and false positive rate, especially for detecting DoS and Probe attacks where the accuracy could reach close to $90 \%$, outperforming the other methods presented in the study, such as a combination of SVM and Rocchio Bundling. The scheme was tested on the 1998 DARPA data set from the MIT Lincoln Lab [31].

Mishra et al. implemented a supervised classification model for anomaly based intrusion detection. The model used principal component analysis for dimensionality reduction and support vector machines to improve the attack detection rate and the computational time. The scheme was implemented on the UNSW-NB15 data set and received a prediction accuracy of $99.9 \%$ for both normal traffic and attacks, as well as a false positive rate of $0.0047 \%$ for normal traffic and $0.0027 \%$ for malicious traffic [11].

\section{Genetic algorithm for intrusion detection}

Balajinath and Raghavan proposed a genetic machine learning algorithm (Genetic algorithm Based Intrusion Detector GBID) as an intrusion detection algorithm. By using 3-tuples consisting of Match index, Entropy index, and Newness index to describe user behavior, the algorithm achieved an accuracy rate of $96.8 \%$ with a false positive rate of $3.2 \%$ [32].

Benaicha et al. implements a genetic algorithm for anomaly based intrusion detection. The algorithm is applied to the NSLKDD data set and achieves a prediction accuracy of $99.74 \%$ and a false positive rate of $3.74 \%$ [33].

\section{Intrusion detection with Naive Bayes and feature reduction}

Mukherjee et al. conducted a study using the Naive Bayes Classifier algorithm for intrusion detection on the NSL-KDD data set, analyzing the same attack types as those analyzed in our study: Probe, DoS, $u 2 r$, and $r 2 l$ ). Multiple feature reduction methods are compared in order to propose the Feature
Vitality Based Reduction Method (FVBRM). Our study only utilizes a single feature reduction method, and therefore a similar comparison with Tree Augmented Naive Bayes would be of future interest. The scheme received up to $97.78 \%$ classification accuracy, with a relatively equal accuracy for DoS attacks (98.7\%), probes (98.8\%), r2l (96.1\%), and normal traffic (97\%). U2R received the lowest accuracy (64\%) [34]. a) Tree Augmented Naive Bayes (TAN) in Intrusion Detection: Several studies also exist where TAN has been applied as an intrusion detection method, such as [12], [13], and [14]. They are consistent in giving promising results but do not highlight the problems of using methods, such as 10-fold cross validation, on the same artificially generated data set, in comparison to using real world generated data.

Najafi et al. performs a similar study as the one presented in this article, but where 10-fold cross validation of the NSL-KDD data set is compared between Naive Bayes, Tree Augmented Naive Bayes, Decision Trees, and Support Vector Machines. The study concludes that Naive Bayes and TAN are less time consuming than support vector machines and decisions trees. The precision and false positive rate is somewhat similar, but TAN performs slightly better than the alternatives, with an average precision of $99.1 \%$ and an average false positive rate of $0.6 \%$. The average precision for the other algorithms were $97.7 \%$ (SVM), 92.1\%, and 99\% (DT). The average false positive rate for the other algorithms were $3.3 \%$ for Naive Bayes, $0.6 \%$ for decision trees, and $1.9 \%$ for Support Vector Machines [13].

Panigrahi et al. also used a Tree Augmented Naive Bayes classifier to detect malicious traffic in the NSL-KDD data set. Three entropy based and three statistical based feature selection methods were used for selection of important features. The study concludes that TAN is a promising algorithm for anomaly based intrusion detection, as it received a top detection rate of $99.7646 \%$ and a lowest false positive rate of $0.2792 \%$ [12].

Nia et al. used a combination of two Bayesian Networks and C5.0 structures for anomaly based intrusion detection. A Tree Augmented Naive Bayes classifier was used to to combine the techniques and the solution was tested on the KDD KUP'99 data set (the original version of the NSL-KDD data set). 41 feature selections were utilized, resulting in a detection accuracy of $99.97 \%$ and false positive rate of $0.38 \%$ [14].

\section{METHOD}

Four versions of TAN were implemented. The different versions were the combinations of TAN with smoothing, without smoothing, using a minimum probability, and not using a minimum probability. The versions were then applied to the NSL-KDD and UNSW-NB15 data sets, and the accuracy, detection rate, and false positive rate were calculated for the classifications of each version. All versions were implemented on the same machine. 


\section{A. Data sets}

All tests on the NSL-KDD data set were run in two classification settings, binary and generic attack types. As described in Section II-B, the binary classifications were "normal" or "intrusion" and the generic attack types were DoS, Probe, $r 2 l$, and $u 2 r$. The UNSW-NB15 data set was also used in two classification settings, binary (normal/malicious) or normal and nine attack categories: Reconnaissance, Backdoor, DoS, Exploits, Analysis, Fuzzers, Worms, Shellcode, and Generic.

Furthermore, many values in the data sets were continuous (such as source bytes and number of failed logins) while the algorithm requires discrete values. To counter this, the values were discretized using the mdlp method proposed by de Sá et al. in [22] and tested by Friedman et al. in [15].

To be able to train and test the algorithms, the NSL-KDD and UNSW-NB15 data sets were divided into training and test sets. While UNSW-NB15 only used the pre-defined training and test sets, NSL-KDD also generated training sets for 10fold cross validation. These generated sets were derived from the pre-defined NSL-KDD training set. By including 10-fold cross validation, the results can more easily be compared with other papers that use different algorithms, such as the plain Naive Bayes classifier.

\section{B. Implementation of algorithm}

The TAN implementations were written in Python 3.8. Except from basic Python modules such as "math, random and copy", the only external library or code used were the scikitlearn library "sklearn.feature_selection", which was used for implementing chi2 feature selection.

TAN was first implemented without the smoothing operator. As expected, TAN without smoothing performed bad. Initially, a lowest conditional probability value was introduced to counter the large amounts of zero frequencies. This lower bound made it possible for the program to compare the probabilities between multiple classifications that contained zero frequencies. By keeping the lower bound to an arbitrary low value, a classification without zero frequencies should still always have a higher classification score than a classification with zero frequencies. This modification is referred to as a "min_p" value.

As expected, the introduced $\min \_p$ modification drastically improved the performance of the TAN implementation (even without the smoothing operator). Subsequently, TAN was implemented with the smoothing operator. A combined version consisting of both the min_p variation and smoothing was also implemented.

Lastly, a feature selection algorithm was implemented. The $K$ best attributes (in terms of their chi2 score) for each training data set and algorithm were selected. Unlike the rest of the implementations, the feature selection was not implemented from scratch but instead obtained from the scikit-learn library.
The $K$ value was approximated by testing the performance with discrete values 5 to 35 with a granularity of 5 .

\section{RESULT}

In this Section the results are presented. Section V-B presents the classification results over the pre-defined test and training sets of the NSL-KDD data set. Both for the binary classification (malicious and normal traffic) and the classification of the generic attack types of the data set (dos, probe, r21, and $\mathrm{u} 2 \mathrm{r}$ ). Section V-C is similar to Section V-B but presents results obtained from ten fold cross validation over the NSLKDD data set, rather than using the inherent train and test sets of the data set. By using 10 fold cross validation, more of the data can be utilized for both training and testing. Section $\mathrm{V}$-D presents the classification results over the pre-defined test and training sets of the UNSW-NB15 data set. Classification results are shown for both binary classification (normal and malicious traffic) and the inherent attack types of the data set (reconnaissance, Backdoor, DoS, Exploits, Analysis, Fuzzers, Worms, and Shellcode).

For the binary classifications, the results are shown for implementation with the smoothing operator (labeled smoothing in the tables ) and no min_p value, with a min_p value and no smoothing operator (labeled $\min \_p$ in the tables), and with neither smoothing nor a min_p value (labeled plain in the tables). In the tables presenting the generic attack types of the data sets, the results are only shown for the implementation using both smoothing and a min_p variable, for brevity. A complete set of all results can be found using the link in Appendix A

\section{A. Evaluation metrics}

The results were analyzed using three traditional metrics: Accuracy, detection rate and false positive rates. The formulas for each metric is given below. In our case, True Positives (TP) are malicious traffic correctly identified as malicious traffic, False Positives (FP) are normal traffic falsely labeled as malicious traffic, True Negatives (TN) are normal traffic labeled as normal traffic, and False Negatives (FN) are malicious traffic labeled as normal traffic.

$$
\begin{gathered}
\text { Accuracy }: \frac{T P+T N}{T P+T N+F P+F N} \\
\text { Recall (detection rate) }: \frac{T P}{T P+F N} \\
\text { False positives }: \frac{F P}{F P+T N}
\end{gathered}
$$

\section{B. NSL-KDD Pre-defined test set}

Table I presents the prediction results of the TAN based intrusion classification over the NSL-KDD data set using binary classification and NSL-KDD's pre-defined test set. The accuracy, detection rate (recall), and false positive rate are 
shown. Three implementations are included for both malicious and normal traffic: TAN with smoothing, TAN with a min_p variable, and TAN without both smoothing and a min_p variable.

\begin{tabular}{|c|c|c|c|}
\hline Traffic & Accuracy & Detection rate & False positives \\
\hline Normal (smoothing) & 0.7782 & 0.9241 & 0.3322 \\
Malicious (smoothing) & 0.7782 & 0.6678 & 0.0759 \\
Normal (min_p) & 0,7810 & 0.9684 & 0.3609 \\
Malicious (min_p) & 0.7810 & 0.6391 & 0.0316 \\
normal (plain) & 0.7355 & 0.4640 & 0.0589 \\
Malicious (plain) & 0.7355 & 0.5466 & 0.0277 \\
\hline
\end{tabular}

PREDICTION RESULTS FROM NORMAL AND MALICIOUS TRAFFIC OF THE NSL-KDD DATA SET

Table II presents the prediction results of the TAN based intrusion classification over the NSL-KDD data set, and displays the result for categorizing normal traffic and the four inherent attack types of the NSL-KDD data set: (dos, probe, $r 2 l$, and $u 2 r$ ). As with table I, the training data is obtained from NSL-KDD's pre-defined test set. The results show the TAN implementation using both smoothing and a min_p variable.

\begin{tabular}{|c|c|c|c|}
\hline Attack type & Accuracy & Detection rate & False positives \\
\hline Normal traffic & 0.8014 & 0.9140 & 0.2839 \\
DoS & 0.9059 & 0.7354 & 0.010 \\
Probe & 0.9253 & 0.6712 & 0.0441 \\
r21 & 0.8534 & 0.1528 & 0.0437 \\
u2r & 0.9728 & 0.2388 & 0.0250 \\
\hline \multicolumn{2}{|c}{ TABLE II }
\end{tabular}

PREDICTION RESULTS FROM THE INHERENT ATTACK TYPES OF THE NSL-KDD DATA SET

\section{NSL-KDD 10-fold cross validation}

Table III presents the prediction results of the TAN based intrusion classification over the NSL-KDD data set using binary classifications and 10-fold cross validation. As with table I, results are shown for TAN with smoothing, TAN with a min_p variable, and TAN without both smoothing and a min_p variable.

\begin{tabular}{|c|c|c|c|}
\hline Type & Accuracy & Detection rate & False positives \\
\hline Normal (smoothing) & 0.9954 & 0.9962 & 0.0055 \\
Malicious (smoothing) & 0.9954 & 0.9945 & 0.0037 \\
Normal (min_p) & 0.9964 & 0.9976 & 0.0050 \\
Malicious (min_p) & 0.9964 & 0.9950 & 0.0024 \\
normal (plain) & 0.9834 & 0.53012 & 0.0032 \\
Malicious (plain) & 0.9834 & 0.9663 & 0.0016 \\
\hline
\end{tabular}

PREDICTION RESULTS FROM NORMAL AND MALICIOUS TRAFFIC OF THE NSL-KDD DATA SET, USING 10-FOLDS CROSS VALIDATION

Table IV presents the prediction results of the TAN based intrusion classification over the NSL-KDD data set, trained and tested using 10-fold cross validation. The results are shown for normal traffic and the inherent attack types of the NSL-KDD data set: (dos, probe, $r 2 l$, and $u 2 r$ ). As with Table II, the results show the TAN implementation using both smoothing and a min_p variable.

\begin{tabular}{|c|c|c|c|}
\hline Attack type & Accuracy & Detection rate & False positives \\
\hline Normal traffic & 0.9930 & 0.9905 & 0.0041 \\
DoS & 0.9945 & 0.9908 & 0.0034 \\
Probe & 0.9952 & 0.9626 & 0.0013 \\
r21 & 0.9948 & 0.9394 & 0.0047 \\
u2r & 0.9972 & 0.8333 & 0.0027 \\
\hline \multicolumn{3}{|c}{ TABLE IV }
\end{tabular}

PREDICTION RESULTS FROM THE INHERENT ATTACK TYPES OF THE NSL-KDD DATA SET, USING 10-FOLDS CROSS VALIDATION

\section{UNSW-NB15 Pre-defined test set}

Table $\mathrm{V}$ presents the prediction results of the TAN based intrusion classifier over the UNSW-NB15 data set using binary classifications (categorizing normal and malicious traffic) and UNSW-NB15's pre-defined test set. The table displays the results with smoothing, a min_p variable, and without both smoothing and a min_p variable.

\begin{tabular}{|c|c|c|c|}
\hline Type & Accuracy & Detection rate & False positives \\
\hline Normal (smoothing) & 0.8780 & 0.9350 & 0.1486 \\
Malicious (smoothing) & 0.8780 & 0.8513 & 0.0650 \\
Normal (min_p) & 0.8797 & 0.9287 & 0.1432 \\
Malicious (min_p) & 0.8797 & 0.8568 & 0.0713 \\
normal (plain) & 0.7904 & 0.4275 & 0.0938 \\
Malicious (plain) & 0.7904 & 0.7086 & 0.0350 \\
\hline
\end{tabular}

PREDICTION RESULTS FROM NORMAL AND MALICIOUS TRAFFIC OF THE UNSW-NB15 DATA SET

Table VI presents the prediction results of the TAN based intrusion classification over the UNSW-NB15 data set. The table shows results for the inherent attack types of UNSBNB15: Reconnaissance, Backdoor, DoS, Exploits, Analysis, Fuzzers, Worms, Shellcode, as well as for normal and generic traffic. The results show the TAN implementation using both smoothing and a min_p variable.

\begin{tabular}{|c|c|c|c|}
\hline Attack type & Accuracy & Detection rate & False positives \\
\hline Normal & 0.88611 & 0.8976 & 0.1193 \\
Reconnaissance & 0.9700 & 0.7129 & 0.0136 \\
Backdoor & 0.9633 & 0.1695 & 0.0287 \\
DoS & 0.8839 & 0.48377 & 0.0860 \\
Exploits & 0.8524 & 0.3535 & 0.0301 \\
Analysis & 0.9626 & 0.1430 & 0.02798 \\
Fuzzers & 0.8954 & 0.3417 & 0.0406 \\
Worms & 0.9980 & 0.1692 & 0.0014 \\
Shellcode & 0.9878 & 0.3319 & 0.0079 \\
Generic & 0.9913 & 0.9819 & 0.0059 \\
\hline
\end{tabular}

PREDICTION RESULTS FROM THE INHERENT ATTACK TYPES OF THE UNSW-NB15 DATA SET

\section{DISCUSSION}

In Section VI-A, the choice to examine and implement TAN is discussed. In Section VI-B, the data sets are discussed. In Section VI-C, the chosen method of measurement is discussed. In Section VI-D, the performance of the different TAN implementations are discussed, as well as their relation to other state-of-the art models for anomaly based intrusion detection 
in computer networks. Lastly, in Section VI-E, potential areas for future work are presented.

\section{A. Choice of Algorithm}

There are many types of algorithms that can be used for anomaly based intrusion detection in computer network environments. Many of these have been extensively tested and some methods are easily available through libraries such as SciKit. Tree Augmented Naive Bayes is not commonly found in such libraries and some implementations that we found contained errors and misunderstandings. Furthermore, there are few satisfactory studies examining TAN as an anomaly based intrusion detection method for computer network traffic. Because of this, TAN was carefully implemented without the use of external resources or libraries. Python 3.8 was used for the implementation as it is common for machine learning and useful for handling matrices and multiple data types. By using Python, the feature reduction was easy to implement, it would also be relatively easy to implement another algorithm from a library such as SciKit.

\section{B. Choice of Data sets}

The NSL-KDD data set was picked because of its extensive use and recognition in other scientific papers. This allows for easier comparison between our results and others. By also running the NSL-KDD data set with 10 -fold cross validation, we can compare our results to the results from studies such as the one by Mukherjee and Sharma [34]. As 10-fold cross validation is run 10 times with $\frac{9}{10}$ of the data set as the training set each time, more of data set is utilized for the training and testing of the data which creates more trustworthy results. Still, in order to better compare our TAN implementation with other algorithms it would be good to implement other models alongside TAN in the same study and using the same machine. this is considered a relevant topic for future research. The UNSW-NB15 data set was chosen to provide more breath and data for the study. It provides a larger training and test set than NSL-KDD and is sampled from more modern data.

\section{Choice of measurement}

As mentioned in Section III, Bhuyan et al. emphasized the importance of measuring the prediction accuracy and false positive rates of intrusion detection models, in order to best gauge the performance of the model and to be able to compare it with other models [26]. We have chosen the same metrics for evaluation. By measuring the accuracy and detection rate of each model, we are able to see how big fractions of intrusions the model is able to detect. A model with high accuracy and detection rate will have a higher chance of detecting an intrusion. It is also important for intrusion detection systems to have a low rate of false positives. An intrusion detection model with a high false positive rate risks disrupting a service by interfering with legitimate traffic.

\section{Model Performance}

As discussed in Section III, many studies have been made on AI-enhanced anomaly based intrusion detection. The prediction accuracy is generally quite high, with some state-of-the-art models reaching above 99\% ( [10] [11] [12]).

TAN is generally no exception, as can be seen in [13] (detection rate of up to $99.0 \%$ and false positive rate of $0.9 \%$ ), [12] (detection rate of $99.8 \%$ and false positive rate of $0.28 \%$ ), [14] (detection rate sometimes reaching $100 \%$, and generally being above 99\%), and as further validated by our study (detection rate of up to $99.8 \%$ and a false positive rate of $0.5 \%$ ). However, when implementing TAN for anomaly based intrusion detection, some factors must be considered in order to achieve an ideal performance.

TAN without smoothing or min_p performs poorly, even compared to how Naive Bayes has performed in other studies, such as the study by Mukherjee and Sharma [34]. This can be explained by zero frequencies. If we do not use smoothing or min_p, the last conditional probability in equation 1 is equal to the frequency of $a_{i}^{\text {test }}$ in entries where both $C=c$ and $\operatorname{parent}\left(A_{i}^{\text {test }}\right)=\operatorname{parent}\left(a_{i}^{\text {test }}\right)$. If this specific entry does not occur in the training set, that specific classification can never be classified with the specific values $a_{i}^{\text {test }}$ and $\operatorname{parent}\left(a_{i}^{\text {test }}\right)$, no matter what the rest of the attributes in the entry is set to. This is a known problem and thus, it is essential to use TAN with a smoothing operator.

This was the case for the tested intrusion data set, as TAN without smoothing performed far worse than TAN with smoothing. More research is required to confirm whether the zero frequency problem is of higher or lower impact for intrusion data sets, than for other types of data sets.

The min_p values increased the performance over all data sets in comparison to all methods without smoothing or a min_p value. This is expected as the min_p value reduces the impact of zero frequencies. This in turn allows for comparison between different classifications even if the latter conditional probability in equation 1 equals to zero for each possible classification.

As this is a well known phenomenon, the standard version of TAN includes smoothing. Therefore, TAN with min_p is not necessarily an improvement of the default TAN and neither does it create a lower time complexity. Both methods run in polynomial time and the only real difference is how to calculate the latter conditional probability in equation 1 . However, it proves a key point in understanding TAN and why smoothing is considered standard.

It can be seen that min_p increases the accuracy of TAN in Section V and Appendix A, and even outperforms TAN with smoothing in some cases. For the individual attack categories among the NSL-KDD data set, there is some recurrence in that TAN with smoothing and without the min_p value outperforms the cases with min_p values without smoothing, such as for the 
the NSL-KDD smurf attack, (0,98 vs 0,57), the NSL-KDD r21 $(0,25 \mathrm{v} 0,02)$, and the NSL-KDD u2r (0,37 vs 0,03$)$. However, far more data is needed to draw conclusions about this. For more information regarding the various results and iterations, see the attached data sheets in Appendix A.

With that said, this paper aims to give insights in the usefulness of TAN classification for anomaly-based intrusion detection in computer networks, as well as provide practical guidelines for what to consider when implementing a TAN classification for such intrusion detection. From the results we can see an overall high accuracy and detection rate as well as a low false positive rate. The performance is considered adequate as it is on par both with other state-of-the-art TAN implementation and with state-of-the-art implementations of other algorithms for networks based anomaly detection. Still, more research is strongly encouraged to investigate the statistical significance of the results, both the results of our TAN algorithm in relation to other TAN implementations for anomaly detection, and for the results of TAN implementations for anomaly detection in relation to the results of other algorithms. Furthermore, more research is encouraged to investigate the statistical significance of the data sets in regards to real cyber attack traffic.

it is also relevant to ask whether the classification accuracy $(99 \%+)$ is enough for anomaly based classification in computer network environments. It could very well be enough, as it seems unfeasible for most computer systems to rely on an intrusion detection rate of $100 \%$. New threats are arising at a regular pace and we continuously see new bugs and vulnerabilities found in systems. Thus, the role of an intrusion detection system should possibly not be to fully remove the risk of attacks, but to act as an assisting tool in an arsenal of other cyber security tools. The IDS should thus aim to detect threats as reliably as possible, while still having other tools and manual investigations to aid the IDS. In this case, a detection rate of $99 \%+$ is a good start. More investigation in this areas is encouraged as future studies. In many of the related studies as well as in our own, the detection rate could be improved by allowing more false positives. For some organizations, a higher false positive rate may be acceptable, for others not. Thus, enterprises could choose what level is best suitable for them, tweaking the parameters to best fit their specific needs. Furthermore, Najafi et al. finds that TAN is almost twice as fast to build as Decision Trees and Support Vector Machines, which gives further validation to TAN's usability for anomaly detection. However, many other suitable algorithms exist and if TAN is to be used, it must be implemented correctly in order to utilize its full performance [13].

\section{E. Future work}

Future research is encouraged on evaluating the ability to adapt on a changing environment, which is one of the strongest arguments for statistically based intrusion detection. To examine this, empirical tests over a longer duration of time would have to be implemented, preferably alongside other non-statistical based intrusion detection models. Another relevant topic for future research is whether an accuracy of $99 \%+$ is enough for intrusion detection in computer network environments. It would also be interesting to compare the performance of attacks with varying severity. Analyzing the CVSS scoring [35] and the NVD data base [36], [37] might be a good way to start such a study. Lastly, further research could be conducted on integrating intrusion detection with threat models and attack simulations [38], [39], or with enterprise architecture models [40], [41].

\section{CONCLUSION}

A Tree Augmented Naive Bayes (TAN) classifier was implemented on two data sets containing simulated data of normal and malicious computer network traffic. TAN displays promising signs of being efficient for the cause, achieving similar performance as the state-of-the-art algorithms, reaching a detection rate and accuracy of well above $99 \%$, while having a false positive rate of $0.5 \%$ or less. However, it is important to implement a smoothing operator when using TAN, or otherwise take action to mitigate the zero frequencies problem. If the implementation does not include a smoothing operator, a min_p value can quickly be implemented to substantially improve the performance of the algorithm. In some cases, the min_p value even outperforms the performance obtained with the smoothing operator. However, there is no evidence for this being more than a coincidence. If neither a smoothing operator nor a min_p value is implemented, TAN will suffer too much from zero frequencies to be effective as an intrusion detection model in computer network environments, and it would be better to use another algorithm for the intrusion detection.

\section{ACKNOWLEDGMENT}

This work has received funding from the Swedish Centre for Smart Grids and Energy Storage (SweGRIDS).

\section{REFERENCES}

[1] N. Perlroth, "Thousands of microsoft customers may have been victims of hack tied to china," The New York Times, 2021.

[2] A. Nair and C. Reese, "Meatpacker jbs says it paid equivalent of $11 \mathrm{~m}$ usd in ransomware attack," Reuters, 2021.

[3] W. Turton and K. Mehrotra, "Meatpackerhackers breached colonial pipeline using compromised password," Bloomberg, 2021.

[4] A. Schiller, J. Binkley, D. Harley, G. Evron, T. Bradley, and C. Willems, Botnets. Burlington: Syngress, 2007.

[5] J. S. Aidan, H. K. Verma, and L. K. Awasthi, "Comprehensive survey on petya ransomware attack," in 2017 International Conference on Next Generation Computing and Information Systems (ICNGCIS), 2017, pp. $122-125$.

[6] R. Lagerström, C. Baldwin, A. MacCormack, D. Sturtevant, and L. Doolan, "Exploring the relationship between architecture coupling and software vulnerabilities," in Engineering Secure Software and Systems, E. Bodden, M. Payer, and E. Athanasopoulos, Eds. Cham: Springer International Publishing, 2017, pp. 53-69.

[7] J. P. Anderson, "Computer security threat monitoring and surveillance," Technical Report, James P. Anderson Company, 1980.

[8] O. Depren, M. Topallar, E. Anarim, and M. Ciliz, "An intelligent intrusion detection system (ids) for anomaly and misuse detection in computer networks," Expert Systems with Applications, vol. 29, no. 4, pp. 713-722, 2005. 
[9] R. Samrin and D. Vasumathi, "Review on anomaly based network intrusion detection system," in 2017 International Conference on Electrical, Electronics, Communication, Computer, and Optimization Techniques (ICEECCOT), 2017, pp. 141-147.

[10] L. Zhiqiang, G. Mohi-Ud-Din, L. Bing, L. Jianchao, Z. Ye, and L. Zhijun, "Modeling network intrusion detection system using feedforward neural network using unsw-nb15 dataset," in 2019 IEEE 7th International Conference on Smart Energy Grid Engineering (SEGE). IEEE, 2019, pp. 299-303.

[11] A. Mishra, A. M. Cheng, and Y. Zhang, "Intrusion detection using principal component analysis and support vector machines," in 2020 IEEE 16th International Conference on Control \& Automation (ICCA). IEEE, 2020, pp. 907-912.

[12] A. Panigrahi and M. R. Patra, "Anomaly based network intrusion detection using bayes net classifiers," 2019.

[13] R. Najafi and M. Afsharchi, "Network intrusion detection using tree augmented naive-bayes," in The Third International Conference on Contemporary Issues in Computer and Information Sciences (CICI), 2012, pp. 396-402.

[14] F. Y. Nia and M. Khalili, "An efficient modeling algorithm for intrusion detection systems using c5.0 and bayesian network structures," in 2015 2nd International Conference on Knowledge-Based Engineering and Innovation (KBEI), 2015, pp. 1117-1123.

[15] N. Friedman, D. Geiger, and M. Goldszmidt, "Bayesian network classifiers," Machine Learning, vol. 29, pp. 131-163, 1997.

[16] F. He and X. Ding, "Improving naive bayes text classifier using smoothing methods," in European Conference on Information Retrieval. Springer, 2007, pp. 703-707.

[17] C. Chow and C. Liu, "Approximating discrete probability distributions with dependence trees," IEEE transactions on Information Theory, vol. 14, no. 3, pp. 462-467, 1968.

[18] C. Bielza and P. Larrañaga, "Discrete bayesian network classifiers: A survey," ACM Comput. Surv., vol. 47, no. 1, 2014.

[19] G. Meena and R. R. Choudhary, "A review paper on ids classification using kdd 99 and nsl kdd dataset in weka," in 2017 International Conference on Computer, Communications and Electronics (Comptelix), 2017, pp. 553-558.

[20] N. Moustafa and J. Slay, "Unsw-nb15: a comprehensive data set for network intrusion detection systems (unsw-nb15 network data set)," in 2015 military communications and information systems conference (MilCIS). IEEE, 2015, pp. 1-6.

[21] J. Dougherty, R. Kohavi, and M. Sahami, "Supervised and unsupervised discretization of continuous features," in Machine Learning Proceedings 1995, A. Prieditis and S. Russell, Eds. San Francisco (CA): Morgan Kaufmann, 1995, pp. $194-202$.

[22] C. R. de Sá, C. Soares, A. Knobbe, P. Azevedo, and A. M. Jorge, "Multi-interval discretization of continuous attributes for label ranking,' in Discovery Science, J. Fürnkranz, E. Hüllermeier, and T. Higuchi, Eds. Berlin, Heidelberg: Springer Berlin Heidelberg, 2013, pp. 155-169.

[23] J. McHugh, "Testing intrusion detection systems: A critique of the 1998 and 1999 darpa intrusion detection system evaluations as performed by lincoln laboratory," ACM Trans. Inf. Syst. Secur, vol. 3, no. 4, p. 262-294, 2000.

[24] M. Tavallaee, E. Bagheri, W. Lu, and A. A. Ghorbani, "A detailed analysis of the kdd cup 99 data set," in 2009 IEEE Symposium on Computational Intelligence for Security and Defense Applications, 2009, pp. 1-6.

[25] N. Moustafa and J. Slay, "Unsw-nb15: a comprehensive data set for network intrusion detection systems (unsw-nb15 network data set)," in 2015 Military Communications and Information Systems Conference (MilCIS), 2015, pp. 1-6.
[26] M. H. Bhuyan, D. K. Bhattacharyya, and J. K. Kalita, "Network anomaly detection: Methods, systems and tools," IEEE Communications Surveys Tutorials, vol. 16, no. 1, pp. 303-336, 2014.

[27] V. Hodge and J. Austin, "A survey of outlier detection methodologies," Artificial intelligence review, vol. 22, no. 2, pp. 85-126, 2004.

[28] A. K. Ghosh and A. Schwartzbard, "A study in using neural networks for anomaly and misuse detection." in USENIX security symposium, vol. 99, 1999 , p. 12.

[29] Y. Ding and Y. Zhai, "Intrusion detection system for nsl-kdd dataset using convolutional neural networks," in Proceedings of the 2018 2nd International Conference on Computer Science and Artificial Intelligence, 2018, pp. 81-85.

[30] D. Elsner, P. Aleatrati Khosroshahi, A. D. MacCormack, and R. Lagerström, "Multivariate unsupervised machine learning for anomaly detection in enterprise applications," in Proceedings of the 52nd Hawaii International Conference on System Sciences, 2019.

[31] L. Khan, M. Awad, and B. Thuraisingham, "A new intrusion detection system using support vector machines and hierarchical clustering," The VLDB journal, vol. 16, no. 4, pp. 507-521, 2007.

[32] B. Balajinath and S. Raghavan, "Intrusion detection through learning behavior model," Computer Communications, vol. 24, no. 12, pp. 1202 $-1212,2001$

[33] S. E. Benaicha, L. Saoudi, S. E. B. Guermeche, and O. Lounis, "Intrusion detection system using genetic algorithm," in 2014 Science and Information Conference. IEEE, 2014, pp. 564-568.

[34] S. Mukherjee and N. Sharma, "Intrusion detection using naive bayes classifier with feature reduction," Procedia Technology, vol. 4, p. $119-128,2012$

[35] P. Johnson, R. Lagerström, M. Ekstedt, and U. Franke, "Can the common vulnerability scoring system be trusted? a bayesian analysis," IEEE Transactions on Dependable and Secure Computing, vol. 15, no. 6, pp. 1002-1015, 2016.

[36] W. Xiong, M. Gülsever, K. M. Kaya, and R. Lagerström, "A study of security vulnerabilities and software weaknesses in vehicles," in Nordic Conference on Secure IT Systems. Springer, 2019, pp. 204-218.

[37] M. Välja, M. Korman, and R. Lagerström, "A study on software vulnerabilities and weaknesses of embedded systems in power networks," in Proceedings of the 2nd Workshop on Cyber-Physical Security and Resilience in Smart Grids, 2017, pp. 47-52.

[38] W. Xiong and R. Lagerström, "Threat modeling-a systematic literature review," Computers \& security, vol. 84, pp. 53-69, 2019.

[39] P. Johnson, R. Lagerström, and M. Ekstedt, "A meta language for threat modeling and attack simulations," in Proceedings of the 13th International Conference on Availability, Reliability and Security, 2018, pp. $1-8$.

[40] M. Österlind, P. Johnson, K. Karnati, R. Lagerström, and M. Välja, "Enterprise architecture evaluation using utility theory," in 2013 17th IEEE International Enterprise Distributed Object Computing Conference Workshops. IEEE, 2013, pp. 347-351.

[41] M. Välja, R. Lagerström, M. Ekstedt, and M. Korman, "A requirements based approach for automating enterprise it architecture modeling using multiple data sources," in 2015 IEEE 19th International Enterprise Distributed Object Computing Workshop. IEEE, 2015, pp. 79-87.

\section{APPENDIX}

The code of the implementation, alongside a more detailed review of the data obtained from the various iterations in the study can be found here: https://github.com/KTH-SSAS/ TAN-intrusion-detection/. 